\title{
ENDOVASCULAR INTERVENTIONS IN PATIENTS WITH MULTILEVEL STENO-OCCLUSIVE LESIONS OF THE INFRARENAL AORTIC ARTERIES
}

\author{
Vasyl Shaprynskyi \\ State Institution of Science «Research and Practical Center \\ of Preventive and Clinical Medicine» State Administrative Department \\ 5 Verhnya str., Kyiv, Ukraine, 01014 \\ Volodymyr Shaprynskyi \\ Department of Surgery No. $1^{1}$ \\ Vasylysa Suleimanova $\bowtie$ \\ Department of Surgery No. $1^{1}$ \\ surgery1@vnmu.edu.ua \\ ${ }^{1}$ National Pirogov Memorial Medical University \\ 56 Pirogova str., Vinnytsya, Ukraine, 21018
}

$\triangle$ Corresponding author

\begin{abstract}
Peripheral artery diseases are a worldwide medical and social problem. Approximately $30 \%$ of patients with critical limb ischemia will undergo amputations and $25 \%$ will die after 1 year. These patients require reconstructive and angioplastic interventions to preserve the limb.

The aim of the study is to compare the effectiveness of different endovascular surgical technologies in patients with multilevel steno-occlusive lesions of the infrarenal aortic arteries.

Materials and methods. Among 243 endovascular interventions, 51 patients were presented with multilevel steno-occlusive lesions of the infrarenal aortic arteries as a result of atherosclerosis obliterans. 42 patients (82.4\%) had two-level lesions and $9(17.6 \%)$ had three-level lesions.

Results. Among 42 patients with a two-level lesion, 29 (56.9\%) ones had femoral arterial segment affection in combination with tibial artery affection. 13 (25.5\%) patients suffered from the lesion of the iliac segment in combination with the affection of the femoral segment arteries. In 9 (17.6 \%) patients the lesions of three or more levels were determined: an iliac segment in combination with femoral and popliteal arteries - in 4 patients, and in 2 patients there were lesions of the femoral, popliteal and tibial segments, in 3 patients there was a lesion of the iliac, femoral, popliteal and tibial-foot segments in different degrees of severity.

7 balloon angioplasties and 13 stenting procedures were performed in two and three-level lesions in which the iliac arterial segment was affected. Only balloon angioplasty was used to revascularize the infraingvinal arterial segments. In the early postoperative period, the complications included thrombosis appeared in 6 patients. It was possible to restore the blood flow and save the limb only in 3 persons. For the other 3 ones, the attempts to restore the patency of the arteries were unsuccessful and resulted in the amputation of the lower extremity. Mortality in the early postoperative period was $3.9 \%$ ( 2 death due to myocardial infarction).

Conclusions. The persons with multilevel steno-obstructive lesions of the infrarenal aortic arteries are the most difficult category of patients with CLI. The surgical method of choice for patients with multilevel steno-occlusive lesions is the endovascular angioplasty with or without stenting.
\end{abstract}

Keywords: atherosclerosis obliterans, revascularization, balloon angioplasty, endovascular intervention, critical limb ischemia.

DOI: 10.21303/2504-5679.2021.002193

\section{Introduction}

Peripheral artery diseases are a worldwide medical and social problem. Lower extremity peripheral artery disease ranks third place among atherosclerotic cardiovascular morbidity, right after coronary events and stroke [1]. For example, 202 million people were affected with peripheral artery diseases in 2010 [2]. The disease prevalence shows the range of $3 \%$ to $10 \%$, increasing to $15 \%$ to $20 \%$ in people over 70 years old [3]. From 2000 to 2010 the number of these patients increased 
by $28.7 \%$ in low-income countries and by $13.1 \%$ in high-income countries [4]. It depends on many factors: aging of the population as demographic trends, genetic and dietary disturbances, smoking, diabetes mellitus can lead to critical leg ischemia [5, 6]. It is strongly associated with concomitant coronary and cerebrovascular diseases. Male dominant over female in the prevalence of disease incidence. Unfortunately, it seems to be increasingly common in the future [4, 7]. The clinical signs and complications of peripheral artery diseases lead to severely reduced life quality. The 5-year mortality rate for these individuals is higher than those of ones with cardiovascular or cerebrovascular disease $[8,9]$. From $1 \%$ to $2 \%$ of persons with atherosclerotic lesions of lower extremities suffer from critical limb ischemia (CLI) as the most severe form of the peripheral arterial disease [2, 10]. The main reason for difficult CLI is multilevel peripheral arterial lesions [11]. Critical limb ischemia leads to leg ischemic rest pain including skin lesions as ulceration or gangrene $[12,13]$. Approximately $30 \%$ of them will undergo amputations and $25 \%$ will die after 1 year [14]. CLI is a surgical problem usually. It means the use of a variety of revascularization techniques to increase distal blood flow and reduce the risk of amputation $[2,11]$. These patients need reconstructive and angioplastic interventions to preserve the limb. The problem «Is the open surgical or endovascular therapy better for patients with CLI?» must be solved individually. But nowadays mini-invasive endovascular interventions have the leading healing trend $[15,16]$. Therefore, CLI is strongly associated with important morbidity, disablement, and mortality. It attracted public attention to find the best strategies for optimum treatment and disease prevention [2, 3]. Recent researches reported about the improvement of the outcomes for patients with CLI [17]. This gives hope to improve the quality of life for such patients and reduce the mortality and amputation rate finally.

The aim of the study is to compare the effectiveness of different endovascular surgical technologies in patients with multilevel steno-obstructive lesions of the infrarenal aortic arteries.

\section{Materials and methods}

This study was conducted at State Institution of Science «Research and Practical Center for Preventive and Clinical Medicine» Kyiv, Ukraine, from January 2014 to January 2021.

During this period 420 patients with confirmed occlusive-stenotic lesions of the infrarenal aortic arteries were admitted to our hospital. Of 420 patients, 243 (57.86 \%) underwent endovascular surgery. We analyzed endovascular surgery for multilevel combined steno-occlusive lesions of the arteries of the lower extremities. Among 243 persons, 51 (20.98\%) patients suffered from multilevel lesions. 42 patients $(82.4 \%$ ) had two-level lesions and 9 (17.6\%) had three-level lesions. According to the Rutherford classification, patients were divided as follows: with category 4 $7(13.7 \%)$, with category $5-32(62.8 \%)$, category 6 was observed in $12(23.5 \%)$ ones [18, 19$]$.

Before entering the patient in the study protocol, a written voluntary agreement to participate in this study was obtained in accordance with the WMA Declaration of Helsinki - Ethical Principles for Medical Research Involving Human Subjects. The study protocol was approved by the Local Ethics Committee (LEC) of the State Institution of Science «Research and Practical Center for Preventive and Clinical Medicine», protocol No. 24 dated 23.11.2013. All patients provided written informed consent.

All patients underwent standard diagnostic procedures using ultrasound of the arteries in the preoperative period.

The operative access and technique were standard in most cases. The endovascular approach was performed under local infiltrative anesthesia and intravenous sedation. Endovascular interventions in multilevel occlusive-stenotic lesions of the infrarenal aortic arteries were determined by anatomical conditions and the degree of CLI in each individual case.

Stages of the surgery. The puncture of the artery was performed according to Seldinger's method and an introducer was implanted [20].

The implantation of the introducer was made under ultrasound navigation (even in distal retrograde access). It let minimize the numerous artery punctures, and thus reducing injuries of the artery walls, avoiding dissection and post-puncture hematomas. The heparin solution was injected through the introducer in a dose of 5000. The next step was to perform arteriography to determine the degree and length of stenotic-occlusive lesion of the artery and the anatomical conditions of revascularization. The 0.035 or 0.014 Guidewire was used to recanalize the occlusive-stenotic 
segment of the lesion. The distal part of the Guidewire was positioned into the relatively passage artery. After inserting the Guidewire through the affected segment, control arteriography was performed to determine the diameter and length of the balloon catheter for angioplasty. In half of the cases, the affected segment was predilated with a smaller diameter balloon to prevent dissection. Final angioplasty was performed with balloons comparable to the size of the artery for 3 minutes. Residual stenosis or signs of arterial wall dissection due to severe calcification was noted in 13 patients, so stents were implanted in this affected area.

After introducer removing, $90 \%$ of patients underwent manual hemostasis (compression) of the puncture point for 3 minutes per 1 French (Fr). For example, if the introducer number was $5 \mathrm{Fr}$, the compression lasted $15 \mathrm{~min}$. The compression roller was appliqued on the skin. In $10 \%$ of patients (especially with obesity) the closing of the puncture area required a special closure device. After the intervention, patients continued to take clopidogrel $75 \mathrm{mg}$ per day in combination with 75 or $100 \mathrm{mg}$ of aspirin daily for 6 months, followed by antiaggregant monotherapy.

For a better understanding, we illustrate a clinical case of endovascular surgical treatment of multi-level steno-occlusive lesions of the left femoral-popliteal-tibial arterial segment using the cross-over technique (because of the stenotic lesion in the proximal part of the superficial femoral artery) in a patient who previously underwent stenting of the superficial femoral artery (Fig. 1).

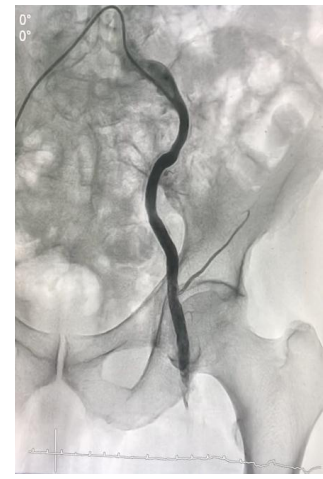

$a$

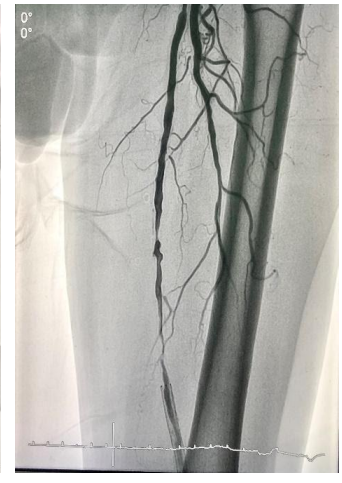

$b$

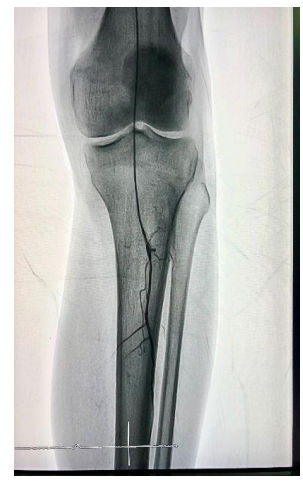

$c$

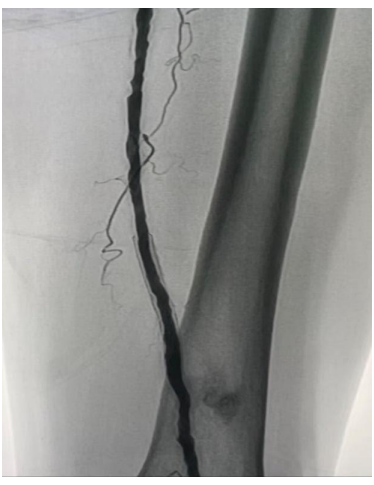

$d$

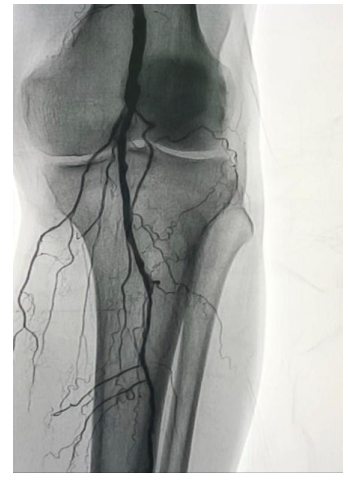

$e$

Fig. 1. Conducting a Guidewire with a catheter according to the cross-over type method due to stenosis in the proximal part of the superficial femoral artery on the left: $a$-Catheter angiography of the left iliac segment; $b$ - Catheter angiography of the left femoral segments; $c$-Catheter angiography of the left popliteal and tibial segments; $d$-Percutaneous

in-stent balloon angioplasty result after catheter balloon angioplasty in the proximal part of the superficial femoral artery; $e$ - Percutaneous balloon angioplasty result in the popliteal artery and the trifurcation area (peroneal artery)

\section{Results}

Among the 42 patients with a two-level lesion, 29 (56.9\%) ones had femoral arterial segment affection in combination with tibial artery affection. In this case, ipsilateral access was used in 19 patients (including 4 distal retrograde access). And in 10 patients the surgical access was used contralateral, due to the proximal level of damage of the superficial femoral artery. It makes impossible the position of the introducer.

In $13(25.5 \%)$ patients the iliac segment lesion in combination with the atherosclerotic affection of the femoral segment arteries was discovered. In this case, 7 patients underwent contralateral access. Brachial access was used on 2 patients due to bilateral lesions of the iliac segments. In 4 patients we performed endovascular intervention only on the single floor (on the segment of inflow arteries).

In $9(17.6 \%)$ patients the lesions of three or more levels were determined. Among them an iliac segment in combination with femoral and popliteal arteries - in 4 patients (in 2 cases contralateral access was used, two more patients underwent ipsilateral access in combination with contralateral puncture access), in 2 individuals femoral, popliteal and tibial lesions of arteries were noted (access ipsilateral), in 3 patients there were lesions of the iliac, femoral, popliteal, and tibial- 
foot segments in different degrees of severity (ipsilateral access in combination with contralateral puncture was used).

7 balloon angioplasties and 13 stenting procedures were performed in two and three-level lesions in which the iliac arterial segment was affected. Only balloon angioplasty was used to revascularize the infranguinal arterial segments.

In some cases, with multi-level combined lesions of the iliac and infrainguinal arterial segments, we performed an endovascular intervention on only one floor (on the segment of the inflow arteries). In the case of the clinical improvement, regression of ischemic manifestations on the limb, we did not perform the second stage of endovascular intervention. If the ischemic symptoms persisted or the regression of ischemia was too slow after discharge of the patient from the hospital, the second operative intervention was revascularization in an endovascular or open way.

In the early postoperative period, the complications included thrombosis appeared in 6 patients. It was possible to restore the blood flow and save the limb only in 3 persons. For the other 3 ones the attempts to restore the patency of the arteries were unsuccessful and resulted in the amputation of the lower extremity.

Mortality in the early postoperative period was $3.9 \%$ ( 2 patients died due to myocardial infarction).

\section{Discussion}

Nowadays there is a lot of unresolved issues and challenges in the treatment of CLI with multilevel obliteration as a significant medical problem. The long total obliterated segments of the arteries tend to have multiple and multilevel lesions. In this case, the surgeon is more likely to use alternative access and advanced re-entry techniques [8, 12].

Thus, the final aim of revascularization must be an overall decline in amputation rates in patients with CLI. But we have to take into account the world trend that the population continues to age, and diabetes mellitus looks like a worldwide epidemic. These factors increase the number of patients with CLI. Of course, they suffer from many comorbidities limiting their ability to tolerate long-time interventions. From this point of view, the use of endovascular interventions is more advantageous. The next question about the advantages or disadvantages of open surgical bypass over endovascular balloon interventions is still open. The same is right for discussion about the preferred approach to treat total occlusions - contralateral or ipsilateral on the lower extremities, antegrade, retrograde, or dual accesses (for example, tibial retrograde and ipsilateral), preferred crossing device, etc. Recent researchers reported that the surgical approaches for CLI depend on the different technics (open or mini-invasive) accepted in various medical centers. It is impossible to believe, but single studies still report about absence of endovascular procedures for CLI [10, 12]. But the bigger part of the authors supports the percutaneous transluminal balloon angioplasty with optional bailout stenting for below-the-knee arterial lesions [14, 19].

According to our results, we widely use endovascular interventions in more than half $(57.86 \%)$ of patients with multilevel steno-occlusive lesions of the infrarenal aortic arteries. Every fourth investigated person in our research suffered from multilevel lesions as a result of CLI. All our patients underwent balloon angioplasty including 13 stenting procedures for the iliac arterial segment. We are deeply convinced, it is better to start revascularization from the endovascular approach. Our opinion supports the fact of the low number of amputations (3 or $6 \%$ ) and mortality rate $-3.9 \%$. Of course, the precise surgical approach must be chosen individually.

Study limitations. The study included all patients older than 40 years old with the diagnosis of CLI from January 2014 to January 2021. The diagnosis of CLI confirmed through ultrasound investigation and clinical picture.

The further research plan includes the study of different types of modern endovascular procedures on arteries depends on multilevel steno-occlusive lesions of the infrarenal aortic arteries.

\section{Conclusions}

Thus, the persons with multilevel steno-occlusive lesions of the infrarenal aortic arteries are the most difficult category of patients with CLI. 
It can be explained with disturbed anatomical conditions, difficult ischemic manifestations in the affected arterial segments, various comorbidities and a high level of complications and mortality.

These patients require simultaneous restoration of blood flow in the arteries of inflow and outflow in different segments with different techniques depends on individual anatomical features of the arteries to determine the optimal puncture access.

The surgical method of choice for patients with multilevel steno-occlusive lesions of the infrarenal aortic arteries is the endovascular angioplasty with or without stenting.

\section{Conflict of interests}

The authors declare that they have no conflicts of interest.

\section{Financing}

The study was performed without financial support.

\section{References}

[1] Fowkes, F. G. R., Rudan, D., Rudan, I., Aboyans, V., Denenberg, J. O., McDermott, M. M. et. al. (2013). Comparison of global estimates of prevalence and risk factors for peripheral artery disease in 2000 and 2010: a systematic review and analysis. The Lancet, 382 (9901), 1329-1340. doi: http://doi.org/10.1016/s0140-6736(13)61249-0

[2] Almasri, J., Adusumalli, J., Asi, N., Lakis, S., Alsawas, M., Prokop, L. J. et. al. (2018). A systematic review and meta-analysis of revascularization outcomes of infrainguinal chronic limb-threatening ischemia. Journal of Vascular Surgery, 68 (2), $624-633$. doi: http://doi.org/10.1016/j.jvs.2018.01.066

[3] Norgren, L., Hiatt, W. R., Dormandy, J. A., Nehler, M. R., Harris, K. A., Fowkes, F. G. R. (2007). Inter-Society Consensus for the Management of Peripheral Arterial Disease (TASC II). European Journal of Vascular and Endovascular Surgery, 33 (1), S1-S75. doi: http://doi.org/10.1016/j.ejvs.2006.09.024

[4] Criqui, M. H., Aboyans, V. (2015). Epidemiology of Peripheral Artery Disease. Circulation Research, 116 (9), $1509-1526$. doi: http://doi.org/10.1161/circresaha.116.303849

[5] Catalano, M. (1993). Epidemiology of critical limb ischaemia: north Italian data. The European journal of medicine, 2 (1), 11-14.

[6] Kröger, K., Stang, A., Kondratieva, J., Moebus, S., Beck, E. et. al. (2006). Prevalence of Peripheral Arterial Disease - Results of the Heinz Nixdorf Recall Study. European Journal of Epidemiology, 21 (4), 279-285. doi: http://doi.org/10.1007/s10654-006-0015-9

[7] Teraa, M., Conte, M. S., Moll, F. L., Verhaar, M. C. (2016). Critical Limb Ischemia: Current Trends and Future Directions. Journal of the American Heart Association, 5 (2). doi: http://doi.org/10.1161/jaha.115.002938

[8] Schürch, K. A., Sixt, S., Jeanneret, C., Canova, C. R. (2018). Update über die aktuelle Diagnostik und Therapie der peripheren arteriellen Verschlusskrankheit (pAVK). Therapeutische Umschau, 75 (8), 478-488. doi: http://doi.org/10.1024/ 0040-5930/a001028

[9] Zeller, T., Baumgartner, I., Scheinert, D., Brodmann, M., Bosiers, M., Micari, A. et. al. (2014). Drug-Eluting Balloon Versus Standard Balloon Angioplasty for Infrapopliteal Arterial Revascularization in Critical Limb Ischemia: 12-Month Results From the IN.PACT DEEP Randomized Trial. Journal of the American College of Cardiology, 64 (15), 1568-1576. doi: http:// doi.org/10.1016/j.jacc.2014.06.1198

[10] Menard, M. T., Farber, A. (2014). The BEST-CLI trial: a multidisciplinary effort to assess whether surgical or endovascular therapy is better for patients with critical limb ischemia. Seminars in Vascular Surgery, 27 (1), 82-84. doi: http://doi.org/ 10.1053/j.semvascsurg.2015.01.003

[11] Dinoto, E., Pecoraro, F., Cutrupi, A., Bracale, U. M., Panagrosso, M., Bajardi, G. (2020). Single staged hybrid approach for multilevel aortic-iliac-femoral-popliteal disease. International Journal of Surgery Case Reports, 77, S166-S169. doi: http://doi.org/10.1016/j.ijscr.2020.09.018

[12] Dominguez, A., Bahadorani, J., Reeves, R., Mahmud, E., Patel, M. (2015). Endovascular therapy for critical limb ischemia. Expert Review of Cardiovascular Therapy, 13 (4), 429-444. doi: http://doi.org/10.1586/14779072.2015.1019472

[13] Laird, J. R., Katzen, B. T., Scheinert, D., Lammer, J., Carpenter, J., Buchbinder, M. et. al. (2012). Nitinol Stent Implantation vs. Balloon Angioplasty for Lesions in the Superficial Femoral and Proximal Popliteal Arteries of Patients With Claudication: Three-Year Follow-up From the RESILIENT Randomized Trial. Journal of Endovascular Therapy, 19 (1), 1-9. doi: http://doi.org/10.1583/11-3627.1

[14] Jens, S., Conijn, A. P., Koelemay, M. J. W., Bipat, S., Reekers, J. A. (2014). Randomized Trials for Endovascular Treatment of Infrainguinal Arterial Disease: Systematic Review and Meta-analysis (Part 2: Below the Knee). European Journal of Vascular and Endovascular Surgery, 47 (5), 536-544. doi: http://doi.org/10.1016/j.ejvs.2014.02.012 
[15] Tepe, G., Laird, J., Schneider, P., Brodmann, M., Krishnan, P., Micari, A. et. al. (2015). Drug-Coated Balloon Versus Standard Percutaneous Transluminal Angioplasty for the Treatment of Superficial Femoral and Popliteal Peripheral Artery Disease. Circulation, 131 (5), 495-502. doi: http://doi.org/10.1161/circulationaha.114.011004

[16] Bradbury, A. W., Adam, D. J., Bell, J., Forbes, J. F., Fowkes, F. G. R., Gillespie, I. et. al. (2010). Bypass versus Angioplasty in Severe Ischaemia of the Leg (BASIL) trial: A survival prediction model to facilitate clinical decision making. Journal of Vascular Surgery, 51 (5), 52S-68S. doi: http://doi.org/10.1016/j.jvs.2010.01.077

[17] Abu Dabrh, A. M., Steffen, M. W., Undavalli, C., Asi, N., Wang, Z., Elamin, M. B. et. al. (2015). The natural history of untreated severe or critical limb ischemia. Journal of Vascular Surgery, 62 (6), 1642-1651.e3. doi: http://doi.org/10.1016/j.jvs.2015.07.065

[18] Amann-Vesti, B. (2013). Scores und Stadien für die Praxis - Angiologie. Therapeutische Umschau, 70 (10), $567-571$. doi: http://doi.org/10.1024/0040-5930/a000448

[19] Rosenfield, K., Jaff, M. R., White, C. J., Rocha-Singh, K., Mena-Hurtado, C., Metzger, D. C. et. al. (2015). Trial of a PaclitaxelCoated Balloon for Femoropopliteal Artery Disease. New England Journal of Medicine, 373 (2), 145-153. doi: http://doi.org/ 10.1056/nejmoa1406235

[20] Hiramatsu K. (1983). Rinsho hoshasen. Clinical radiography, 28 (11 Suppl), 1127-1135.

How to cite: Shaprynskyi, V., Shaprynskyi, V., Suleimanova, V. (2021). Endovascular interventions in patients with multilevel steno-occlusive lesions of the infrarenal aortic arteries. EUREKA: Health Sciences, 6, 24-29. doi: http://doi.org/10.21303/ 2504-5679.2021.002193 